\title{
Extra Yukawas: Searching for New Scalars via Triple-Top Signature
}

\section{Tanmoy Modak*广}

Department of Physics, National Taiwan University, Taipei 10617, Taiwan

E-mail: tanmoyy@hep1.phys.ntu.edu.tw

\begin{abstract}
The study of top quark is one of the major program at LHC. Essentially being a top factory, LHC provides a unique opportunity to study multi-top productions. In the Standard Model (SM), the single-top, $t \bar{t}$ and four-top production cross sections are very large and well over pb level, while the triple-top is few fb. Any enhancement in triple-top production would be intriguing. This may be realized in the Two-Higgs-Doublet-Model (2HDM) without a discrete $Z_{2}$ symmetry, where both the doublets couple to the up- and down-type quarks. After diagonalization of the fermion mass matrices, two different Yukawas $\kappa_{f}$ and $\rho_{f}$ emerge, where $\kappa_{f}$ is diagonal and real, however, $\rho_{f}$ is in general non-diagonal and complex. Extra Yukawas $\rho_{t t}$ and $\rho_{t c}$ with $\mathscr{O}(1)$ magnitude can enhance the cross section of triple-top process to $\mathrm{pb}$ level. In this proceeding we discuss the discovery potential of new scalars via two novel signatures: $c g \rightarrow t H^{0} / t A^{0} \rightarrow t t \bar{c}$ (same-sign top) and $c g \rightarrow t H^{0} / t A^{0} \rightarrow t t \bar{t}$ (triple-top) at LHC. Discovery may shed light on the Baryon Asymmetry of the Universe.
\end{abstract}

The 39th International Conference on High Energy Physics (ICHEP2018)

4-11 July, 2018

Seoul, Korea

*Speaker.

${ }^{\dagger}$ Work is done in collaboration with Masaya Kohda and Wei-Shu Hou. 


\section{Introduction}

The discovery [1] of the $125 \mathrm{GeV}$ scalar $\left(h^{0}\right)$ has opened up the search for additional scalar doublet that may exist in nature. The SM like nature of $125 \mathrm{GeV}$ scalar indicates the approximatealignment phenomena. This might imply that the second doublet is heavy i.e. the approximatealignment from decoupling. However, approximate-alignment may emerge without decoupling $[2,3,4]$, allowing sub-TeV second doublet. This brings the search for pseudo-scalar $A^{0}$ and CPeven scalar $H^{0}$ well within the reach of LHC. Approximate-alignment without decoupling can also be realized in the $2 \mathrm{HDM}$ without discrete $Z_{2}$ symmetry [5]. The $2 \mathrm{HDM}$ without discrete $Z_{2}$ symmetry opens up the possibility of extra Yukawas such as $\rho_{t c}$ and $\rho_{t t}$. It was recently discussed [6] that $\rho_{t t}$ and $\rho_{t c}$ with $\mathscr{O}(1)$ magnitude and complex phase can drive electroweak baryogenesis (EWBG) efficiently. Together, $\rho_{t c}$ and $\rho_{t t}$ induce two clean processes $c g \rightarrow t A^{0} / t H^{0} \rightarrow t t \bar{c}$ and $c g \rightarrow t A^{0} / t H^{0} \rightarrow t t \bar{t}$ at LHC. Focusing in the range $350 \mathrm{GeV} \leq m_{A^{0}}\left(m_{H^{0}}\right) \leq 700 \mathrm{GeV}$, we discuss the discovery potential of $p p \rightarrow t A^{0} / t H^{0}+X \rightarrow t t \bar{c}+X$ and $p p \rightarrow t A^{0} / t H^{0}+X \rightarrow t t \bar{t}+X$ followed by leptonic decays of all top quarks. The discovery of any of these two processes would help us understand mechanism behind EWBG.

\section{Framework and Results}

For our study, the relevant extra Yukawa couplings for the up-type quarks are [7],

$$
\frac{\rho_{i j}}{\sqrt{2}} \bar{u}_{i L}\left(H^{0}+i A^{0}\right) u_{j R}+\text { h.c. }
$$

where, $\rho_{i j}$ non-diagonal complex matrix, but plausibly [5] shares the same "flavor organization" features of SM, i.e. trickling down of off-diagonal elements as reflected in the observed quark masses and mixings. Therefore, we expect $\rho_{t t}$ and $\rho_{t c}$ to be $\mathscr{O}\left(\kappa_{t}\right)$, where $\kappa_{t} \sim \sqrt{2} m_{t} / v(v \approx$ $246 \mathrm{GeV}$ ) is the top Yukawa in SM. Similarly, $\rho_{b b}$ and $\rho_{\tau \tau}$ (and $\rho_{\tau \mu}$ ) most likely be $\sim \kappa_{b}$ and $\sim \kappa_{\tau}$ respectively. Note that $\rho_{c t} \simeq 0$ is demanded by $B$ physics constraints [8,9]. Here, we took $\cos \gamma \rightarrow 0$ for simplicity, where $\cos \gamma$ [5] is the mixing angle between the CP-even scalars $h^{0}$ and $H^{0}$ (analogous to $\cos (\beta-\alpha)$ in $2 \mathrm{HDM}$ II).
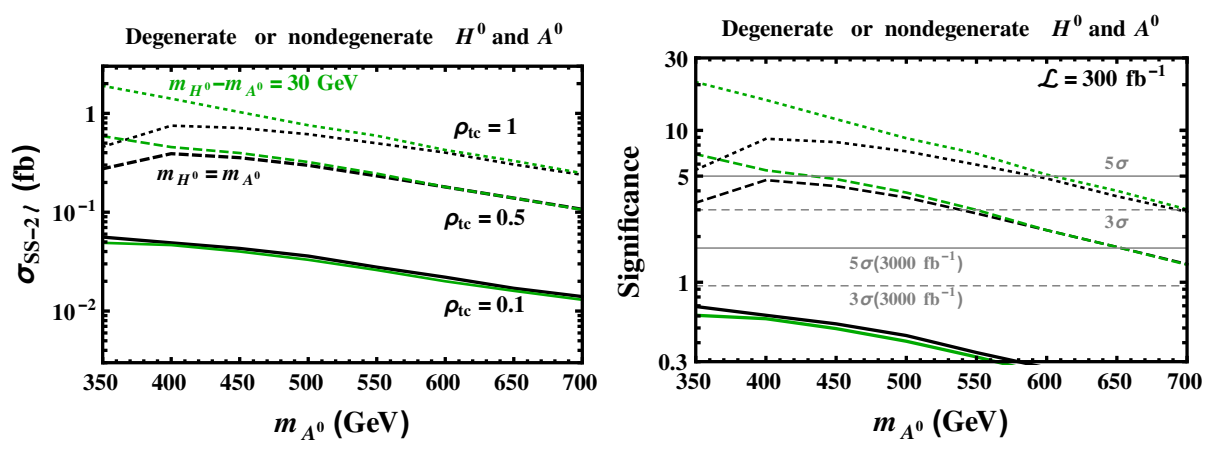

Figure 1: Signal cross sections (left) and discovery potential (right) of SS2 $\ell$ for $m_{H^{0}}=m_{A^{0}}$ (black) and $m_{H^{0}}-m_{A^{0}}=30 \mathrm{GeV}$ (light green), with $\rho_{t c}=1,0.5,0.1$, setting $\rho_{t t}=1$. The CM energy and integrated luminosity chosen for the analysis are $\sqrt{s}=14 \mathrm{TeV}$ and $300 \mathrm{fb}^{-1}$ respectively. 

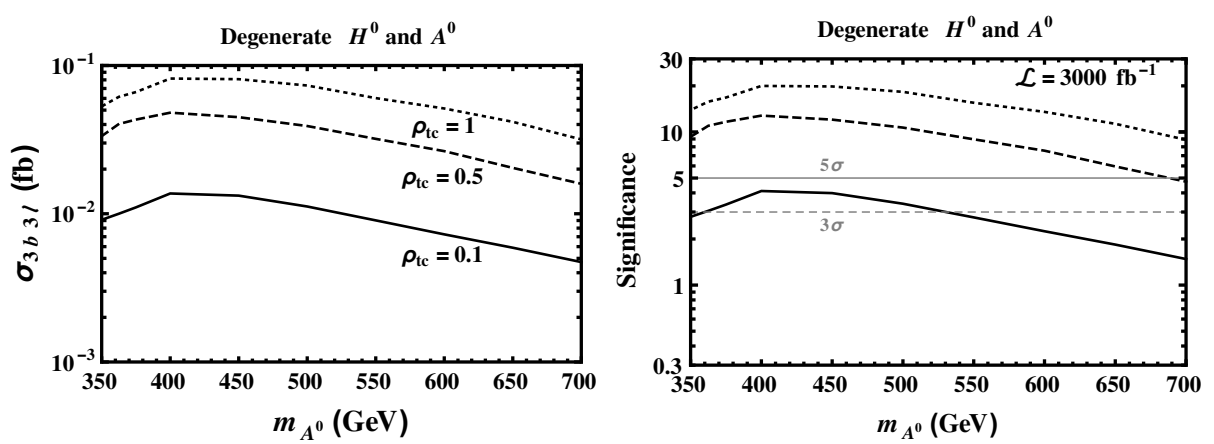

Figure 2: The signal cross sections (left) and discovery potential (right) of $3 b 3 \ell$ process for $m_{A^{0}}=m_{H^{0}}$ with $\rho_{t c}=1,0.5,0.1$, setting $\rho_{t t}=1$ at $14 \mathrm{TeV}$ LHC. The integrated luminosity is chosen to be $3000 \mathrm{fb}^{-1}$.

Same-sign top.- The same-sign top process solely depends on the flavor changing neutral Higgs coupling $\rho_{t c}$. In order to demonstrate the discovery potential of $p p \rightarrow t A^{0} / t H^{0}+X \rightarrow t t \bar{c}+X$ process, we look for leptonic decays of both the top quarks and select events with two same sign leptons $(e$ and $\mu)$, at least three jets with at least two of them are $b$-tagged. We denote this final state as SS2 $\ell$. The SM background processes considered are: $t \bar{t} Z, t \bar{t} W, t Z+$ jets, $3 t+j, 3 t+W$, $4 t$, and $t \bar{t} h$. The $t \bar{t}$ and $Z / \gamma^{*}+$ jets processes may contribute if the charge of one of the leptons get misidentified. The largest background is non-prompt contribution [10], which is about 1.5 times of the second largest background $t \bar{t} W^{1}$. Note that, the $p p \rightarrow t A^{0}+X \rightarrow t t \bar{c}+X$ and $p p \rightarrow t H^{0}+X \rightarrow$ $t t \bar{c}+X$ processes interfere destructively if masses and widths of $H^{0}$ and $A^{0}$ are degenerate. The cancellation effect alleviates when $m_{H^{0}}$ and $m_{A^{0}}$ and/or $\Gamma_{H^{0}}$ and $\Gamma_{A^{0}}$ become non-degenerate (see Refs. $[11,12]$ for details). In this scenario, the SS2 $\ell$ cross section becomes the incoherent sum of $p p \rightarrow t A^{0}+X \rightarrow t t \bar{c}+X$ and $p p \rightarrow t H^{0}+X \rightarrow t t \bar{c}+X$ processes. In Fig. 1, we summarize the signal cross sections and discovery potential for $\rho_{t c}=1,0.5$, and 0.1 setting $\rho_{t t}=1$. In order to illustrate the impact of this cancellation effect, we chose two different mass hierarchies for $H^{0}$ and $A^{0}: m_{H^{0}}=m_{A^{0}}$ (black contours) and $m_{H^{0}}-m_{A^{0}}=30 \mathrm{GeV}$ (green contours). As non-zero $\rho_{t t}$ makes $\Gamma_{H^{0}} \neq \Gamma_{A^{0}}$, the cancellation between $H^{0}$ and $A^{0}$ cross sections are not exact. Hence the cross sections do not vanish for $m_{H^{0}}=m_{A^{0}}$ case, as can be seen from black lines in Fig. 1 (left). For $m_{H^{0}}-m_{A^{0}}=30$ case both masses and widths become non-degenerate, resulting in green lines with larger cross sections. It is clear from Fig. 1 that discovery might happen for wide range of parameter space. E.g. if $\rho_{t c}=1$ and $350 \mathrm{GeV} \lesssim m_{A^{0}} \lesssim 600 \mathrm{GeV}$ discovery is possible with $\mathscr{L}=300 \mathrm{fb}^{-1}$ dataset for the $m_{H^{0}}-m_{A^{0}}=30$ scenario. We discovery contours for full HL-LHC data $\left(\mathscr{L}=3000 \mathrm{fb}^{-1}\right)$ are also shown in the right panel of Fig. 1 . We find if $\rho_{t c}=0.1$, discovery is beyond even for full HL-LHC data.

Triple top.- The triple-top process $c g \rightarrow t A^{0} / t H^{0} \rightarrow t t \bar{t}$ is more exquisite and depend on both $\rho_{t c}$ and $\rho_{t t}$ couplings. To determine the discovery probability $p p \rightarrow t A^{0} / t H^{0}+X \rightarrow t t \bar{t}+X$, we focused on the final state where all three top quarks decay leptonically. The signature constitutes at least three leptons, at least three jets of which at least three are $b$-jets, and $E_{T}^{\text {miss }}$ (denoted as $3 b 3 \ell$ ). Although in the SM the triple-top cross section is about few fb [14], there exist several

\footnotetext{
${ }^{1}$ For the details of the cut based analysis, background cross sections and QCD correction factors see Ref. [11]. The correction factors for $Z / \gamma^{*}+$ jets processes can be found in Refs. [13].
} 
other SM backgrounds which contribute to the overall background cross section. The dominant SM backgrounds are $t \bar{t} Z+$ jets and $4 t$, while $t \bar{t} W b, t Z j b, 3 t+W$, and $t \bar{t} h$ conform subdominant contributions. The $t \bar{t}+$ jets process can contribute if a jet gets misidentified as a lepton. The details of the cut based analysis and QCD correction factors for different backgrounds can be found in Ref. [11]. The cross sections for $3 b 3 \ell$ are smaller due to finer selection cuts than SS2 $\ell$, hence, we give results for $3000 \mathrm{fb}^{-1}$. Unlike the same-sign top, the $A^{0}$ and $H^{0}$ contributions do not cancel each other for triple-top. Hence, in Fig. 2, we only show the cross sections and discovery potential for $m_{H^{0}}=m_{A^{0}}$ (see Ref. [11] for other mass hierarchies). We can see from Fig. 2 [right] that $5 \sigma$ (or higher) significance is possible if $m_{A^{0}} \lesssim 700 \mathrm{GeV}$ and even for $\rho_{t c} \gtrsim 0.5$. Unlike SS2 $\ell$, if $\rho_{t c} \sim 0.1$, one could still get some hint (at $3 \sigma$ or higher) up to $m_{H^{0}}=m_{A^{0}} \sim 500 \mathrm{GeV}$ with $3000 \mathrm{fb}^{-1}$ dataset. Hence, we advocate that the "triple-top" search is more exquisite and informative.

\section{Summary}

The $2 \mathrm{HDM}$ without a discrete $Z_{2}$ symmetry allows the possibility of extra Yukawas $\rho_{t t}$ and $\rho_{t c}$. The $\rho_{t t}, \rho_{t c}$ couplings induce two novel signatures at LHC: same-sign top $\left(c g \rightarrow t H^{0} / t A^{0} \rightarrow t t \bar{c}\right)$ and triple-top $\left(c g \rightarrow t H^{0} / t A^{0} \rightarrow t t \bar{t}\right)$. The former process may emerge in the early part of Run 3, while the triple-top process may require HL-LHC data. Discovery would enable us understand the origin of matter-antimatter asymmetry of the Universe. We also remark that the recent discovery of $t \bar{t} h$ provides additional probe for $\rho_{t t}$ driven EWBG [15].

\section{References}

[1] G. Aad et al. [ATLAS Collaboration], Phys. Lett. B 716, 1 (2012); S. Chatrchyan et al. [CMS Collaboration], ibid. B 716, 30 (2012).

[2] J. F. Gunion and H. E. Haber, Phys. Rev. D 67, 075019 (2003).

[3] Carena et al., JHEP 1404, 015 (2014).

[4] Bechtle et al., Eur. Phys. J. C 77, 67 (2017).

[5] W.-S. Hou and M. Kikuchi, Eur. Phys. Lett. 123, 11001 (2018).

[6] K. Fuyuto, W. S. Hou and E. Senaha, Phys. Lett. B 776, 402 (2018).

[7] See, e.g., S. Davidson and H.E. Haber, Phys. Rev. D 72, 035004 (2005).

[8] B. Altunkaynak et al., Phys. Lett. B 751, 135 (2015).

[9] A. Crivellin, A. Kokulu and C. Greub, Phys. Rev. D 87, 094031 (2013).

[10] A.M. Sirunyan et al. [CMS Collaboration], Eur. Phys. J. C 77, 578 (2017).

[11] M. Kohda, T. Modak and W. S. Hou, Phys. Lett. B 776, 379 (2018).

[12] W. S. Hou, M. Kohda and T. Modak, Phys. Lett. B 786, 212 (2018).

[13] W. S. Hou, M. Kohda and T. Modak, Phys. Rev. D 96, 015037 (2017); W. S. Hou, M. Kohda and T. Modak, Phys. Rev. D 98, 015002 (2018); M. Kohda, T. Modak and A. Soffer, Phys. Rev. D 97, 115019 (2018).

[14] V. Barger, W.-Y. Keung and B. Yencho, Phys. Lett. B 687, 70 (2010).

[15] W. S. Hou, M. Kohda and T. Modak, Phys. Rev. D 98, 075007 (2018). 